\title{
Relative Discrepancy in Social Dilemma Games: the Utility of the Prisoner's Dilemma Game in the Social Sciences and Humanities
}

\author{
Gregg D. Bromgard ${ }^{1}$, David Trafimow \\ Department of Social Sciences Southern University at New Orleans \\ New Mexico State University600 Press Drive New Orleans, LA 70006
}

\begin{abstract}
This paper demonstrates how social bargaining games can be used to study a broad range of study areas the prisoner's dilemma," basic methodological instructions, and a sample study to demonstrate the utility of the prisoner's dilemma game in the social sciences and humanities. We examined participants' emotional responses to situations that produce conflict. We manipulated outcomes within the prisoner's dilemma by creating three sub-outcomes within the "sucker's payoff" outcome. Each sub-outcome had different payoff structures and participants were asked to imagine that they were the victims of the other player's strategy to defect. We posit three possible hypotheses why participants should feel anger toward the other player: (1) the other player violated the participants' autonomy by defecting; (2) the participants felt frustrated because the other player blocked the participants' goal of attaining money, and (c) the participants perceived the payoffs as unfair relative to the other player. We obtained results that supported the hypothesis that participants perceived the payoffs as unfair.
\end{abstract}

Keywords: Prisoner's Dilemma, Relative Deprivation, Anger, "Shadenfreude." within the social sciences and humanities. This paper includes a general overview of the social dilemma game called.

\section{INTRODUCTION}

The prisoner's dilemma game is a resourceful utility that can be employed in all the social sciences and humanities. In this article I describe an actual study that was conducted. This paper serves as an advertisement to all the social sciences and humanities on how simple manipulations in the payoff structure (discussed below) can produce interesting and intriguing implications that can be interpreted from a broad range of fields in the social sciences and humanities. My hope is that researchers who do not usually collect quantitative data or researcher who do not use human participants in a formal experiment can use the prisoner's dilemma game - and manipulated payoff structures - to produce data within each of their respective fields. This article include empirical data to demonstrate how manipulating the payoff structure of the prisoner's dilemma game can produce interesting results. The current experiment manipulated outcomes within the prisoner's dilemma by creating three sub-outcomes within the "sucker's payoff" outcome. Each sub-outcome had different payoff structures and participants were asked to imagine that they were the victims of the other player's strategy to defect. We posit three possible hypotheses why participants should feel anger toward the other player: (1) the other player violated the participants' autonomy by defecting; (2) the participants felt frustrated because the other player blocked the participants' goal of attaining money, and (c) the participants perceived the payoffs as unfair relative to the other player.

\section{RELATIVE DiSCREPANCY IN SOCIAL DILEMMA GAMES}

Situations that produce anger are of importance in our everyday lives. In light of the recent protests regarding immigration policies in Germany (Turkish immigrants), France (Albanian immigrants), and the United States (Hispanic immigrants), the current study focuses on situations that elicit anger and can, perhaps, lead to action (such as protests, demonstrations, acts of aggression and rebellions). People become angry and aggressive for various reasons, many of which are unknown to observers. Several researchers explain anger and characterize situations that elicit anger with elaborate experiments, noisy questionnaires or esoteric models (Rozin, Lowery, Haidt, \& Imada, 1999; Dollard, Doob, Miller, Mowrer, \& Sears, 1939; Gurr, 1970). The current study is an attempt to integrate and simplify the previous research on situations that elicit anger with situations created from social bargaining games.

1 Corresponding Author: gbromgard@suno.edu 


\section{American Research Journal of Humanities and Social Sciences, Volume 1, Issue1, Feb-2015}

ISSN 2378-7031

Our goal is to identify situations, using the prisoner's dilemma game, that elicit anger while manipulating or controlling factors such as moralistic violations, frustration, and relative discrepancies.

\section{Situations that Produce Anger}

\subsection{Anger in Response to Moral Violations}

Human beings and primates seem especially sensitive to violations of social order when witnessing an act that violates another human being or primate (de Waal, 1996). This sensitivity often results in emotional reactions, which have been found to be good predictors of moral judgements (Haidt, Koller, \& Dias, 1993; Shweder, Mahapatra, \& Miller, 1987).

Two clusters of emotions have been hypothesized to result from violations of morality. The first cluster of emotions includes shame, embarrassment, and guilt. This cluster of emotions involves assessments of self-worth within the community (Rozin, et al., 1999). The second cluster of emotions, which Izard (1971/1977) refers to as the hostility triad, includes contempt, anger, and disgust. This hostility triad concerns violations of morality by other people.

According to Shweder, Much, Mahapatra, and Park (1997) there are three distinct ethics that cultures use to approach and resolve moral issues. These three ethics include (a) community ethics; (b) autonomy ethics, and (c) divinity ethics. These ethics are based on different conceptualizations of the person. For example, community ethics characterize the person as an office holder within an interdependent community; autonomy ethics represent the person as an individual; and divinity ethics look at the person as a divine creature.

Rozin et. al. (1999) demonstrated that three emotions (contempt, anger, and disgust) map onto violations of these three moral codes. Participants from the United States and Japan read a list of moral code violations and then chose the most appropriate face that they believed an on-looker would make. The situations presented to the participants were moral code violations as described by Shweder et al. (1997). Rozin et al. (1999) found that contempt was elicited by violations of community, anger by violations of autonomy, and disgust by violations of divinity.

\subsection{Anger in Response to Frustration}

Frustration has been demonstrated to be another source of anger (Geen, 1998). Frustration is usually defined as interference with or blocking of the attainment of one's goal. The pioneers of frustration research claimed that frustration tends to arouse aggressive feelings (Dollard, Doob, Miller, Mowrer, \& Sears, 1939). Dollard and colleagues (1939) were the first to develop a unified theory of aggression (i.e., the frustration-aggression hypothesis). Briefly stated, the frustration-aggression hypothesis claims that frustration evokes a state of instigation to aggress, which is a drive state that motivates behavior in the same way as do primary drives like hunger or thirst (Geen, 1998).

Barker, Dembo, and Lewin (1941) performed a classic study that illustrates how frustration is a source of anger. Children observed toys that were inside a room they were not allowed to enter. The children stood outside the room, wanting to play with the toys but were unable to reach them. After some time, the children were finally able to go inside the room. These children smashed the toys against the walls and floors and behaved very destructively. Other children, who were allowed to enter the room and play with the toys without any delay, played in a less destructive way than did the children who were frustrated before playing with the toys.

\subsection{Anger in Response to Relative Deprivation}

According to Gurr (1970), an individual experiences deprivation, or a sense of grievance, only when the individual thinks that it is not feasible to obtain "X." Gurr formulated relative deprivation as value expectations minus value capabilities divided by value expectations.

\section{$\frac{\text { Walue Expectation - Walue Capabilities }}{\text { Walue Expectation }}=$ Relative Deprivation}

"Value expectations" are the goods or opportunities the individual wants and feels entitled to, based on comparisons with similar others. "Value capabilities" are those goods or opportunities the individual possesses.

Crosby (1976) further illustrates five preconditions in order for an individual to feel relative deprivation. The person who lacks "X" must (a) see that someone else possesses "X," (b) want "X," (c) feel entitled to "X," (d) think it feasible to obtain "X," and (e) lack a sense of personal responsibility for not having "X." 


\section{American Research Journal of Humanities and Social Sciences, Volume 1, Issue1, Feb-2015 ISSN 2378-7031}

Both Gurr's and Cosby's models claim that relative deprivation results when there is something person A wants that person B has and that person A feels that some type of injustice has occurred to prevent him/her from having what person B possesses. Firestone (1974), Gurr (1970) and Runciman (1966) argue that revolutions and social violence result from relative deprivation.

\section{THE PRISONER's DILEMMA GAME}

The most common type of social dilemma game is the prisoner's dilemma game, where two individuals (person A and person B) must choose between two strategies: cooperate or defect. This two-person two-strategy game results in four unique outcomes. If both individuals choose to cooperate then the outcome is referred to as the "mutual cooperation" outcome. If both individuals choose to defect, then the outcome is referred to as the "mutual defection" outcome. If person A chooses to cooperate, but person B chooses to defect, then this is referred to as the "sucker's payoff" (from person A's perspective). On the other hand, if person A defects and person B cooperates, then this is referred to as the "temptation to defect" outcome (from person A's perspective).

Each of the four unique outcomes has a unique payoff structure. For example, in the "mutual cooperation" outcome, each person receives $\$ 25.00$. In the "mutual defection" outcome each person receives $\$ 5.00$. In the "sucker's payoff" outcome, the member that defected receives $\$ 50.00$, while the member who cooperated receives nothing. The "temptation to defect" outcome mirrors the "sucker's payoff." Each party is better off choosing to defect rather than cooperate, regardless of what the other person's choice is (for a review of rational strategies see Axelrod, 1984). Yet if both persons choose to defect, both are worse off than if both would have chosen to cooperate. This dilemma is referred to as the "DD trap" (mutual defection trap) by Rapoport and Chammah (1965).

\subsection{Altering the Payoff Structures}

In order to create the aforementioned situations that produce anger (violations of individual rights, frustration, and relative deprivation), the current research restructured the payoffs structure in the prisoner's dilemma game only within the "sucker's payoff" outcome. Three different "sucker's payoff" outcomes were created from the perspective of the other person: (a) the "gain" condition; (b) the "neutral" condition, and (c) the "loss" condition. In the "gain" condition, the opponents receives $\$ 50.00$ while the participant receives nothing; in the "neutral" condition, both participants receive $\$ 1.00$, and in the "loss" condition, the opponent loses $\$ 25.00$ and the participant receives nothing.

The logic of restructuring the payoffs in the "sucker's payoff" outcome follows arguments made by Ferguson and Rule (1983) on situations that produce aggression (and anger). They illustrate three questions a person must ask and answer after being harmed by someone: (1) what was the act that produced the harm? (2) what ought to have been done under the circumstances? and (3) does any discrepancy exist between what was done and what ought to have been done?

The first question raises the issue of the intentionality of the act. If it is found that the intent to hurt was attributed to the other person, a second judgement is made about whether the intention was malevolent. Within the prisoner's dilemma game, the opponent defected against the participant who cooperated, then the opponent's actions are deemed intentional. This is precisely what occurs in the traditional prisoner's dilemma game's "sucker's payoff" outcome and is referred to in the current research as the "gain" condition.

The second and third questions pertain to what ought to have been done under the circumstance and if there are any discrepancies that exist between what was done and what should have been done. These values reflect norms that Ferguson and Rule (1983) claim are determined by culture. Most cultures have norms and laws that condemn and prohibit the infliction of malevolent harm to others. Within the prisoner's dilemma game, it has already been established that if the opponent defected against the participant who cooperated, the opponent's actions are perceived as intentional. However, given a situation where cooperation benefits both players more than any one player defecting, the opponent's action would be deemed malevolent. This was the logic for creating the "neutral" condition.

The logic of the "loss" condition is to examine the participant's emotional reaction when they cooperated while the opponent defected and when the payoffs clearly punish the opponent for defecting. Not only does the defector get punished but the cooperator also suffers by not benefiting. The "loss" condition is different from the "neutral" condition in that (a) the defector is punished and (b) the cooperator receives at least something in the "neutral" condition, whereas in the "loss" condition, the cooperator receives nothing. 


\section{THE CURRENT RESEARCH}

The current study examines the emotions that are elicited from the three payoff sub-outcomes within the "sucker's payoff" outcome in the prisoner's dilemma game. The "sucker's payoff" outcome was only examined because it is the only outcome that is of theoretical interest to the current study. There are three competing hypotheses. First, violations of one's individual rights are predicted to elicit anger. This hypothesis is consistent with Rozin et al.'s (1999) research on moral code violations. Second, the blockage of one's goals are predicted to elicit anger. This is consistent with the aggression-frustration hypothesis proposed by Dollard et al. (1939). Finally, unfair outcomes are predicted to elicit anger. This is consistent with the relative deprivation hypothesis proposed by Gurr (1970).

The present experiment tests the hypothesis that, within the prisoner's dilemma game, it is unfair outcomes that elicit the most anger, not violations of individual rights or frustration. We are not arguing that situations that violate individual rights or situations that produce frustration do not elicit anger, we are arguing that when controlling for these variables, we expect the most anger only in situations where there is relative deprivation. This experiment holds situations that violate individual rights and situations that produce the aggression-frustration hypothesis constant by creating three conditions in which the other player always defects against the participants. That is, in all three conditions, the other player is violating the participants' individual rights and concurrently preventing the participant from obtaining their goal (to win money). This isolates relative deprivation as the only factor that could create differences in emotional responses within each of the "sucker's payoff" sub-outcomes.

In addition, we hypothesize that the opponent's failure to obtain monetary gain at the expense of the participant will elicit "happiness" or "schadenfreude." "Schadenfreude" is a German word that translates into "getting pleasure out of another person's misfortune." We predict that more "schadenfreude" will be elicited in the "neutral" outcome. In this outcome, the opponent's strategy to defect is unsuccessful and as a result of this unsuccessful strategy the opponent receives nothing while the participant receives $\$ 1.00$. The participants are predicted to feel good about the other player's failed strategy; that is, the participants are getting pleasure out of their opponent's misfortune even though the participants only received $\$ 1.00$. "Shadenfreuda" is not predicted in the "gain" condition because the opponent's strategy to defect was successful. In the "loss" outcome, the opponent's strategy was unsuccessful and the opponent is "punished" for defected. "Shadenfreuda" is not predicted for this outcome because (1) the participant may deem the opponent as particularly malevolent because they were willing to withstand a loss to ensure the participant did not receive a gain (the strategy to defect was by "design") and (2) the participant may feel like the opponent is being justly punished because the opponent loses $\$ 25.00$.

\section{Methodology}

\subsection{Participants}

Twenty-two male $(n=7)$ and female $(n=15)$ participants between the ages of eighteen and twenty-three participated for class credit. Most of our sample consisted of participants who self-identified as being either of European or Hispanic ancestry with two African-Americans and one Native American. Participants were randomly assigned to conditions.

\subsection{Materials and Procedure}

The experiment was presented as a survey to participants in a classroom setting. The survey was presented in three parts. The first part instructed participants on how to play the prisoner's dilemma game. The second part of the survey presented standardized universal facial expressions (Ekman \& Matsumoto, 1993a and b) of the following emotions: contempt, happiness, sadness, disgust, fear and anger (see Appendix A). Standardized expressions of surprise, embarrassment and a neutral expression were also included. Participants were instructed to pay careful attention to the facial expressions and were told that they could always refer back to the facial expressions at anytime during the study. The third part of the survey instructed participants that they were to imagine they were going to play the prisoner's dilemma game against a "real" opponent (gender of the opponent was not specified). Next, participants were asked to imagine themselves in the "mutual cooperation" scenario and rate the likelihood of feeling each of the emotions that were previously presented. Participants were then asked to imagine themselves in the "sucker's payoff" scenario and, again, rated the likelihood of feeling each of the emotions. Participants were only presented with the "mutual cooperation" outcome followed by the "sucker's payoff" outcome.

The "sucker's payoff" outcome was manipulated by having three different payoff sub-outcomes. The payoff suboutcomes included: (1) the typical "sucker's payoff structure referred to as the "gain" condition; (2) the "neutral" condition, and (3) the "loss" condition. 


\section{American Research Journal of Humanities and Social Sciences, Volume 1, Issue1, Feb-2015 ISSN 2378-7031}

All participants were asked to imagine the "mutual cooperation" outcome first. This gave participants the opportunity to perceive the actual benefits of cooperating. In all conditions, the "mutual cooperation" outcome payoff was $\$ 25.00$ for each person. Participants were asked to rate the likelihood of feeling each of the nine universal emotional expressions when they imagined themselves in the mutual cooperation outcome. The rating scale was a 6-point scale consisting of the following terms; "Extremely Unlikely" (-3), "Quite Unlikely"(-2), "Slightly Unlikely" $(-1)$, "Slightly Likely" $(+1)$, "Quite Likely" $(+2)$, and "Extremely Likely." $(+3)$. These ratings were later re-coded so that "- 3 " equaled " 0 " and " +3 " equaled " +6 ". Next, participants were asked to imagine that they were in one of the "sucker's payoff" sub-outcomes (when the participant cooperated and the opponent defected) and indicate the likelihood of feeling each of the nine universal emotional expressions.

The "Gain" condition. The "gain" condition is the typical "sucker's payoff" outcome in the prisoner's dilemma game. The dilemma results from participants' temptation to defect because it has the possibility of winning the most money. In this condition, the participant receives nothing for cooperating while the opponent receives $\$ 50.00$ for defecting.

The "Neutral" condition. This condition eliminated any dilemma the players may have had to defect by eliminating the incentive. If both players cooperated (resulting in the mutual cooperation outcome) they would receive $\$ 25.00$. If both players defected (mutual defection outcome) they would receive $\$ 5.00$. If, however, either player chose opposite choices (resulting in the "temptation to defect" or "sucker's payoff" outcome) each player received only $\$ 1.00$. Here, the best strategy was for both players to cooperate.

The "Loss" condition. This condition is identical to the "neutral condition" except that the opponent is punished for defecting by taking away $\$ 25.00$ while the participant, who cooperated, receives nothing. Again, the logical strategy in this condition is for both players is to cooperate.

\section{RESULTS}

We had two hypotheses and therefore used planned contrasts for each hypothesis. The first hypothesis stated that participants would indicate the likelihood of feeling more anger in the "gain" condition than in the "neutral" or "loss" condition. A one-way ANOVA was performed that indicated a significant difference between conditions for the likelihood of feeling "anger," $F(2,19)=4.90, p=.019$. LSD post hoc analyses revealed that anger was significantly greater in the "gain" condition $(\mathrm{M}=4.00$, s.d. $=.63)$ when compared to the "neutral" condition $(\mathrm{M}=$ 2.14 , s.d. $=1.77)(\mathrm{p}=.04)$ and when compared to the "loss" condition $(\mathrm{M}=1.56$, s.d. $=1.67)(\mathrm{p}=.00)$. No other significant effects were obtained (p.'s > .05) (See Table 1).

The second hypothesis stated that participants would indicate the likelihood of feeling more "happiness" or "schadenfreude" in conditions where the opponent's strategy failed. These conditions were the "neutral" and "loss" conditions. A one-way ANOVA was performed and indicated a significant difference between conditions for the likelihood of feeling "happiness" or "schadenfreude," F $(2,19)=3.65, \mathrm{p}=.046$. LSD post hoc analyses revealed that "happiness" or "schadenfreude" was significantly greater in the "neutral" condition $(\mathrm{M}=2.71$, s.d. $=1.25)$ when compared to the "gain" condition $(\mathrm{M}=.83$, s.d. $=.75)(\mathrm{p}=.014)$. However, we did not find any evidence of participants feeling more "happiness" or "schadenfreude" in the "loss" condition $(\mathrm{M}=1.78$, s.d. $=1.48)$ when compared to the "gain" condition (p. =.17). No other significant effects were obtained (p.'s > .05).

Since we had no predictions for the other emotions ("disgust", "embarrassment", "surprise", "fear", "sadness", "neutral", and "contempt"), we conducted a MANOVA and did not obtain any significant effects (all p.'s > .05).

\section{DISCUSSION}

Our study supports the hypothesis that the most anger is elicited by unfair outcomes in the prisoner's dilemma game. This hypothesis is consistent with the literature on relative deprivation (Gurr, 1970). The person who lacks "X" must: (a) see that someone else possesses "X"; (b) want "X"; (c) feel entitled to "X"; (d) think it feasible to obtain "X", and (e) lack a sense of personal responsibility for not having "X." In other words, the participants: (a) saw that the other person had more money; (b) wanted more money; (c) felt entitled to more money; (d) believed it was feasible to obtain more money, and (e) did not hold themselves responsible for not having the money.

Our study provided only weak support for the hypothesis that anger results from violations of individual rights (participants indicated they would feel angry, but not as much as in the "loss" condition). According to Rozin et. al. (1999) particular emotions map onto violations of moral codes. One such violation is an act that violates another person's autonomy or individual rights by defecting. Our study created conditions in which one person could violate 


\section{American Research Journal of Humanities and Social Sciences, Volume 1, Issue1, Feb-2015 ISSN 2378-7031}

another person's autonomy or individual rights. However, we found little evidence that defection alone by the opponent elicited anger. Across all conditions, the opponent defected against the participant, yet it was only when the defection caused unequal payoffs that the participants indicated feeling angry.

We also found little evidence that frustration was the main source of anger in our experiment. In each condition, the participant's goal - to accrue money - was thwarted by the other player. Again, it was only in the condition that had unfair outcomes that the participants indicated they felt anger.

In addition to conditions that elicit anger, we also obtained interesting data on situations that elicit happiness or "schadenfreude." Participants indicated that they were most likely to feel happiness when the opponent's strategy to defect failed. In other words, participants felt happiest when the opponent made a hostile act and this hostile act was trumped by the payoff structure.

Two possible interpretations exist for participants' happiness in "neutral" condition. One interpretation is that the participants simply felt that justice was served. For example, in the "loss" condition, participants may have felt that the opponent was being punished for his/her hostility (the opponent's strategy to defect cost him/her twenty-five dollars). Whereas, in the "neutral" condition, the punishment fit the crime. In addition, participants might have simply felt relieved that no matter what the opponent's motives were, they at least will have benefited a little (i. e., the participant and opponent received \$1.00).

Another interpretation is that participants may have felt a form of "schadenfreude." "Schadenfreude" is a German word that translates into "getting pleasure out of another person's misfortune." The best evidence for "schadenfreude" was found in the "neutral" condition. In this condition, the opponent's strategy to defect was unsuccessful and as a result both players received one dollar. The participants may have felt good about the other player's failed strategy; however, if the opponent suffered too severely (the "loss" condition) the participants were more reluctant to indicate feeling "happiness" or "schadenfreude."

\section{Conclusion, Policy Implications And Limitations}

Our study was an attempt to model real social interactions utilizing the prisoner's dilemma game. Our study has several limitations. We found evidence that anger was elicited from unfair outcomes and these results are interpreted in terms of relative deprivation (Gurr, 1970). However, we did not measure the variables that are proposed by relative deprivation (The person who lacks "X" must: (a) see that someone else possesses "X"; (b) want "X"; (c) feel entitled to "X"; (d) think it feasible to obtain "X", and (e) lack a sense of personal responsibility for not having "X"). Future research should attempt to measure if the participants: (a) saw that the other person had more money; (b) wanted more money; (c) felt entitled to more money; (d) believed it was feasible to obtain more money, and (e) felt that they were not responsible for not having money.

A second limitation in our study is that the prisoner's dilemma game is highly artificial thereby raising questions concerning ecological and external validity. In addition, our participants imagined that they were in these various situations, and they may have responded differently if these situations had been real.

We proposed three hypotheses about how situations that produce conflict elicit anger: (a) violations of individual rights; (b) frustration, and (c) relative deprivation. However, our data were most consistent with the relative deprivation hypothesis. Therefore, relative deprivation should provide fertile ground for future research.

In addition, relative deprivation should also shed light on the current protests over immigration in Germany, France, and the United States. For example, the United States is facing a huge wave of illegal immigration from Mexico and countries in South America. Currently, new legislation is being proposed to thwart illegal immigrants from coming into the United States. These legislation proposals have ignited mass demonstrations and protests from legal and illegal Hispanic immigrants. The protesting can be interpreted with the results of this study. Not only are illegal immigrants frustrated (frustration-aggression hypothesis) and feel their autonomy is threatened, they also feel like (a) there is an opportunity for financial gain in the United States, (b) they are entitled to this financial gain opportunity, (c) it is possible to obtain this financial gain opportunity, and (d) they are not responsible for not having the financial gain opportunity.

Finally, it is the hope of the current research that this study provided a useful demonstration on how all social sciences and humanities can creatively construct situations and manipulated the payoff structures to test their own hypotheses from their own respective fields and interests. 


\section{American Research Journal of Humanities and Social Sciences, Volume 1, Issue1, Feb-2015 ISSN 2378-7031}

\section{REFERENCES}

[1] Axelrod, R. (1984). The evolution of cooperation. New York: Basic Books.

[2] Barker, R. G., Dembo, T., \& Lewin, K. (1941). Frustration and regression: An

[3] experiment with young children. University of Iowa Studies in Child Welfare, 18, (1), 1-314.

[4] Crosby, F. (1976). A model of egoistical relative deprivation. Psychological Review, 83,

[5] 85-113.

[6] de Waal, F. B. (1996). Good natured. Cambridge, MA: Harvard University Press.

[7] Dollard, J., Doob, L., Miller, N. E., Mowrer, O. H., \& Sears, R. (1939). Frustration and

[8] aggression. New Haven, CT: Yale University Press.

[9] Ekman, P., \& Matsumoto, D. (1993a). JACFEE. Japanese and Caucasian facial

[10] expressions of emotions.

[11] Ekman, P. \& Matsumoto, D. (1993b). JACNeuF. Japanese and Caucasian neutral faces.

[12] Ferguson, T. J., \& Rule, B. G. (1983). An attributional perspective on anger and

[13] aggression. In R. G. Geen \& E. Donnerstein (Eds.), Aggression: Theoretical and empirical reviews (Vol. 1: theoretical and methodological issues, pp. 41-74). New York: Academic Press.

[14] Firestone, J. M. (1974). Continuities in the theory of violence. Journal of Conflict

[15] Resolution, 18, 117-142.

[16] Geen, R. G. (1998). Aggression and social behavior. In D. T. Gilbert, S.T. Fisk, \& G.

[17] Lindzey (Eds.), The handbook of social psychology (pp. 317-356). New York: McGraw-Hill.

[18] Gurr, T. R. (1970). Why men rebel. Princeton, NJ: Princeton University Press.

[19] Haidt, J., Koller, S. H., \& Dias, M. G. (1993). Affect, culture, and morality, or is it wrong

[20] to eat your dog? Journal of Personality and Social Psychology, 65, 613-628.

[21] Izard, C. E. (1971). The face of human emotion. New York, NY: Appleton-Century-Crofts.

[22] Izard, C. E. (1977). Human emotions. New York: Plenum Press.

[23] Rapoport, A. \& Chammah, A.M. (1965). Gender differences in factors contributing to the

[24] level of cooperation in the prisoner's dilemma. Journal of Personality and Social Psychology, 2, 831-838.

[25] Rozen, P., Lowery, L., Haidt, J., \& Imada, S. (1999). The CAD hypothesis: A mapping

[26] between three moral emotions (contempt, anger, disgust) and three moral codes (community, autonomy, and divinity). Journal of Personality and Social Psychology, 76, 574-586.

[27] Runciman, W. G. (1966). Relative deprivation and social justice: A study of attitudes to

[28] social inequality in twentieth-century England. Berkeley and Los Angles: Berkeley University Press.

[29] Shweder, R. A., Mahapatra, M., \& Miller, J. (1987). Culture and moral development. In

[30] J. Kagan \& S. Lamb (Eds.), The emergence of morality in young children (pp. 1-83). Chicago: University of Chicago Press.

[31] Shweder, R. A., Much, N. C., Mahapatra, M., \& Park, L. (1997). The "big three" of

[32] morality (autonomy, community, divinity) and the "big three" explanations of suffering. In A. Brandt \& P. Rozen (Eds.), Morality and health (pp. 119-169). New York: Kelly. 
American Research Journal of Humanities and Social Sciences, Volume 1, Issue1, Feb-2015

ISSN 2378-7031

Table1. Means for Likelihood of Emotion

\begin{tabular}{|l|l|l|l|}
\hline & "gain" & "neutral" & "loss" \\
\hline Anger & $4.00^{*}$ & 2.14 & 1.56 \\
\hline & & & \\
\hline Happy & .83 & $2.71^{*}$ & 1.78 \\
\hline
\end{tabular}

Means are significant at $\mathrm{p}<.05$

\section{APPEnDiX A}

Japanese and Caucasian Facial Expressions of Emotion and Japanese and Caucasian Neutral Faces (Ekman \& Matsumoto, 1993
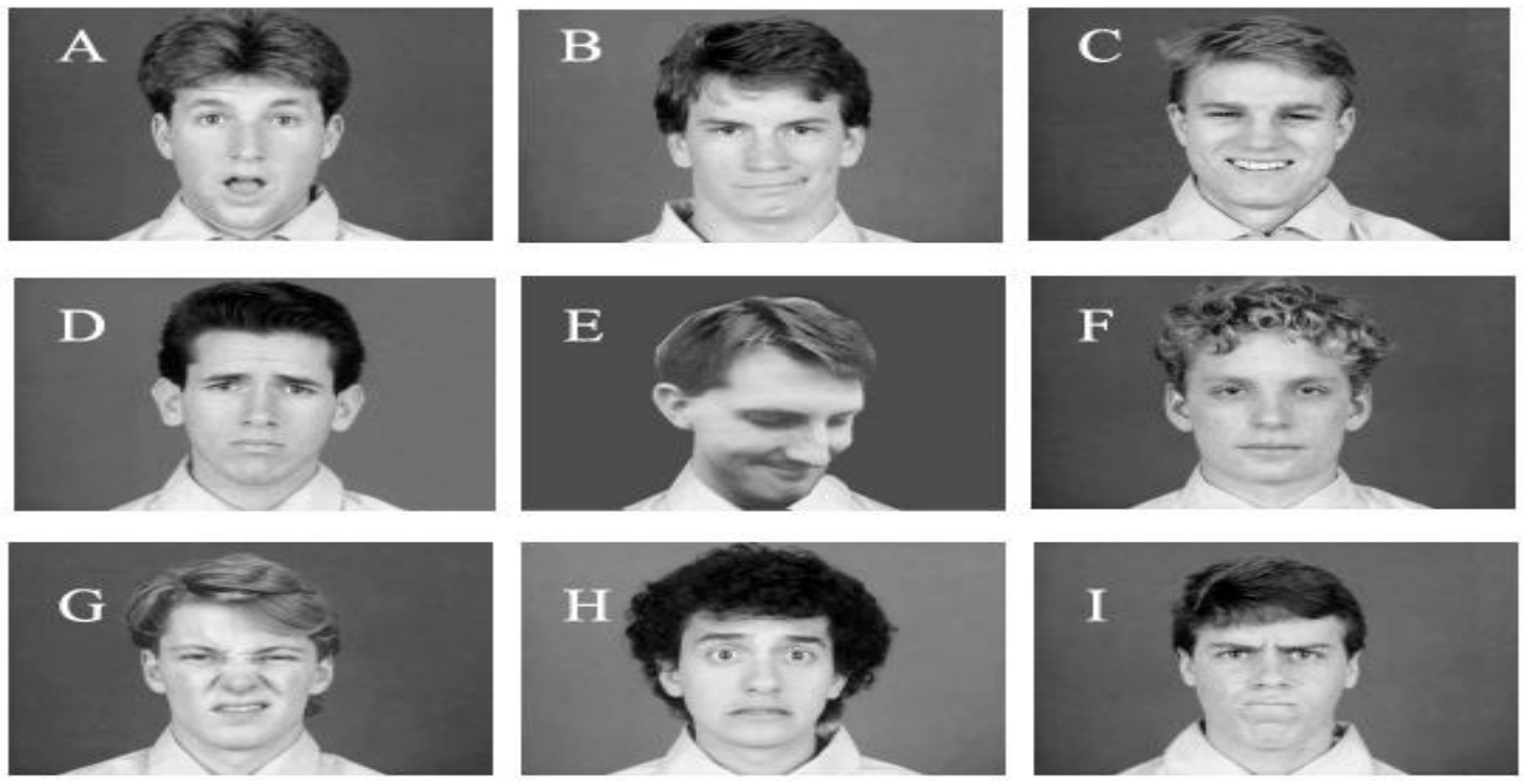